1. P. J. Davis \& P. Rabinowitz, Numerical Integration, Blaisdell, Waltham, Mass., 1967.

2. D. Elliotr, "A Chebyshev series method for the numerical solution of Fredholm integral equations," Comput. J., v. 6, 1963, pp. 102-111. MR 27 \#5386.

3. A. H. Stroud \& D. Secrest, Gaussian Quadrature Formulas, Prentice-Hall, Englewood Cliffs, N. J., 1966. MR 34 \#2185.

\title{
An Explicit Sixth-Order Runge-Kutta Formula
}

\author{
By H. A. Luther
}

1. Introduction. The system of ordinary differential equations considered has the form

$$
d y / d x=f(x, y), \quad y\left(x_{0}\right)=y_{0} .
$$

Here $y(x)$ and $f(x, y)$ are vector-valued functions

$$
\begin{aligned}
y(x) & =\left(y_{1}(x), y_{2}(x), \cdots, y_{m}(x)\right), \\
f(x, y) & =\left(f_{1}(x, y), f_{2}(x, y), \cdots, f_{m}(x, y)\right),
\end{aligned}
$$

so that we are dealing with $m$ simultaneous first-order equations.

For the fifth-order case, explicit Runge-Kutta formulas have been found whose remainder, while of order six when $y$ is present in (1), does become of order seven when $f$ is a function of $x$ alone [3], [4]. This is due to the use of six functional substitutions, a necessary feature when $y$ occurs nontrivially [1].

A family of explicit sixth-order formulas has been described [1]. In this family is the formula given in the next section. Its remainder, while of order seven when $y$ is present in (1), is of order eight when $f$ is a function of $x$ alone. Here again the possibility arises because seven functional substitutions are used, rather than six. Once more, this is a necessity [2].

For selected equations (those not strongly dependent on $y$ ) such formulas seem to lead to some increase in accuracy.

2. Presentation of the Formula. For the interval $\left[x_{n}, x_{n}+h\right]$, Lobatto quadrature points leading to a remainder of order eight are

$x_{n}, \quad x_{n}+h / 2, \quad x_{n}+\left(7-(21)^{1 / 2}\right) h / 14, \quad x_{n}+\left(7+(21)^{1 / 2}\right) h / 14, \quad x_{n}+h$.

A set of Runge-Kutta formulas related thereto is given below. They can be verified by substitution in the relations given by Butcher [1].

Expressed in a usual form they are

Received December 28, 1966. Revised July 31, 1967. 


$$
\begin{gathered}
y_{n+1}=y_{n}+\left\{9 k_{1}+64 k_{3}+49 k_{5}+49 k_{6}+9 k_{7}\right\} / 180 \\
k_{1}=h f\left(x_{n}, y_{n}\right) \\
k_{2}=h f\left(x_{n}+\nu h, y_{n}+\nu k_{1}\right) \\
k_{3}=h f\left(x_{n}+h / 2, y_{n}+\left\{(4 \nu-1) k_{1}+k_{2}\right\} /(8 \nu)\right) \\
k_{4}=h f\left(x_{n}+2 h / 3, y_{n}+\left\{(10 \nu-2) k_{1}+2 k_{2}+8 \nu k_{3}\right\} /(27 \nu)\right) \\
k_{5}=h f\left(x_{n}+\left(7+(21)^{1 / 2}\right) h / 14, y_{n}+\left\{-\left([77 \nu-56]+[17 \nu-8](21)^{1 / 2}\right) k_{1}\right.\right. \\
-8\left(7+(21)^{1 / 2}\right) k_{2}+48\left(7+(21)^{1 / 2}\right) \nu k_{3} \\
\left.\left.\quad-3\left(21+(21)^{1 / 2}\right) \nu k_{4}\right\} /(392 \nu)\right)
\end{gathered}
$$

If desired, a companion formula can be found by replacing $(21)^{1 / 2}$ throughout with $-(21)^{1 / 2}$. The parameter $\nu$ may have any value other than zero.

3. A Choice of Parameter. In some senses, a "best" formula is one for which each coefficient of $k_{i}$ in expressions such as

$$
f\left(x_{n}+h / 2, y_{n}+\left\{(4 \nu-1) k_{1}+k_{2}\right\} /(8 \nu)\right)
$$

is positive or zero. If this is impossible, we may seek to minimize the sum of the absolute values of the coefficients. To establish a figure of merit, this sum should be divided by the weight $1 / 2$ in $x_{n}+h / 2$. In this connection see, for example, [5, p. 146]. The resulting expression for the above, assuming $\nu>0$, is

$$
1 /(4 \nu)+|1-1 /(4 \nu)| \text {. }
$$

This is clearly nonincreasing, and is a minimum of 1 for $\nu \geqq 1 / 4$.

The other components of (2) behave in like manner except for that involving $k_{7}$, which is of the form $a / \nu+b$, where $a$ and $b$ are positive constants. Except for this component, the minimum is achieved for all if $\nu \geqq 4\left(55+9(21)^{1 / 2}\right) / 331>1$.

If the same tactics are applied to the formula resulting when $-(21)^{1 / 2}$ is used rather than $(21)^{1 / 2}$, it develops that all components are minimized if $\nu \geqq 1 / 4$ except that pertaining to $k_{5}$, which is of the form $a / \nu+b, a$ and $b$ positive.*

To determine whether to use the formula pertaining to $(21)^{1 / 2}$, as in $(2)$, or that formed therefrom by replacing $(21)^{1 / 2}$ by $-(21)^{1 / 2}$, we need the actual minima. For $(21)^{1 / 2}$, in the order $k_{2}, k_{3}, k_{4}, k_{5}, k_{6}, k_{7}$, they are

$$
1,1,1,17 / 7,\left(232+33(21)^{1 / 2}\right) / 35,4 /(3 \nu)+\left(526+259(21)^{1 / 2}\right) / 90 \text {. }
$$

For $-(21)^{1 / 2}$, in the same order, they are

$$
1,1,1,4 /(7 \nu)+\left(55+3(21)^{1 / 2}\right) / 28,\left(41(21)^{1 / 2}-13\right) / 28,\left(130+63(21)^{1 / 2}\right) / 18 .
$$

Since one is ideal, a comparison shows (the fundamental weights for $y_{n+1}$ are also to be considered) that $-(21)^{1 / 2}$ is to be preferred, and that, if we desire $0<\nu \leqq 1$, the value of $\nu$ should be one. The resulting $k_{i}$ formulas are

* The author is indebted to the referee for pointing out that the sign of the surd might be used to advantage. 


$$
\begin{aligned}
& k_{1}=h f\left(x_{n}, y_{n}\right) \\
& k_{2}=h f\left(x_{n}+h, y_{n}+k_{1}\right) \\
& k_{3}=h f\left(x_{n}+h / 2, y_{n}+\left\{3 k_{1}+k_{2}\right\} / 8\right) \\
& k_{4}=h f\left(x_{n}+2 h / 3, y_{n}+\left\{8 k_{1}+2 k_{2}+8 k_{3}\right\} / 27\right) \\
& k_{5}=h f\left(x_{n}+\left(7-(21)^{1 / 2}\right) h / 14, y_{n}+\left\{3\left(3(21)^{1 / 2}-7\right) k_{1}-8\left(7-(21)^{1 / 2}\right) k_{2}\right.\right. \\
& \left.\left.+48\left(7-(21)^{1 / 2}\right) k_{3}-3\left(21-(21)^{1 / 2}\right) k_{4}\right\} / 392\right) \\
& k_{6}=h f\left(x_{n}+\left(7+(21)^{1 / 2}\right) h / 14, y_{n}+\left\{-5\left(231+51(21)^{1 / 2}\right) k_{1}\right.\right. \\
& -40\left(7+(21)^{1 / 2}\right) k_{2}-320(21)^{1 / 2} k_{3}+3\left(21+121(21)^{1 / 2}\right) k_{4} \\
& \left.\left.+392\left(6+(21)^{1 / 2}\right) k_{5}\right\} / 1960\right) \\
& k_{7}=h f\left(x_{n}+h, y_{n}+\left\{15\left(22+7(21)^{1 / 2}\right) k_{1}+120 k_{2}\right.\right. \\
& +40\left(7(21)^{1 / 2}-5\right) k_{3}-63\left(3(21)^{1 / 2}-2\right) k_{4} \\
& \left.\left.-14\left(49+9(21)^{1 / 2}\right) k_{5}+70\left(7-(21)^{1 / 2}\right) k_{6}\right\} / 180\right) \text {. }
\end{aligned}
$$

4. Acknowledgement. The particular technique shown was an outgrowth of research supported by the National Aeronautics and Space Administration under Grant NGR-44-001-024.

Department of Mathematics

Texas A\&M University

College Station, Texas 77843

1. J. C. Butcher, "On Runge-Kutta processes of high order," J. Austral. Math. Soc., v. 4, 1964, pp. 179-194. MR 29 \#2972.

2. J. C. Butcher, "On the attainable order of Runge-Kutta methods," Math. Comp., v. 19, 1965, pp. 408-417. MR 31 \#4180.

3. H. A. LUTHER, "Further explicit fifth-order Runge-Kutta formulas," SIAM Rev., v. 8, 1966, pp. 374-380. MR 34 \#3796.

4. H. A. LUTHER \& H. P. Konen, "Some fifth-order classical Runge-Kutta formulas," SIAM Rev., v. 7, 1965, pp. 551-558. MR 32 \#1909.

5. L. D. GATES, JR., "Numerical solution of differential equations by repeated quadratures," SIAM Rev., v. 6, 1964, pp. 134-147. MR 29 \#6626. 